\title{
In situ video-STM studies of the mechanisms and dynamics of electrochemical bismuth nanostructure formation on Au†
}

\author{
H. Matsushima, ta $^{\text {S. }- \text { W. Lin, }}{ }^{\text {b }}$ S. Morin ${ }^{b}$ and O. M. Magnussen*a
}

Received 20th April 2016, Accepted 9th May 2016

DOI: $10.1039 / c 6 f d 00086 j$

\begin{abstract}
The microscopic mechanisms of Bi electrodeposition on $\mathrm{Au}(111)$ and $\mathrm{Au}(100)$ electrodes in the overpotential regime were studied by in situ scanning tunneling microscopy with high spatial and temporal resolution. Atomic resolution images of the needle-like Bi(110) deposits formed on $\mathrm{Au}(111)$ reveal the central influence of covalent $\mathrm{Bi}-\mathrm{Bi}$ bonds on the deposit morphology. In the straight steps along the needle edges the $\mathrm{Bi}$ atoms are interlinked by these bonds, whereas at the needle tip and at kinks along the needle edges dangling bonds exist, explaining the rapid structural fluctuations at these sites. For ultrathin $\mathrm{Bi}$ deposits on $\mathrm{Au}(100)$ a more open atomic arrangement was found within the surface plane, which was tentatively assigned to an epitaxially stabilised Bi(111) film. Furthermore, well-defined nanowires, consisting of zigzag chains of Bi surface atoms, were observed on this surface.
\end{abstract}

\section{Introduction}

Quasi one-dimensional nanostructures, such as nanowires, nanotubes and nanoribbons, have unique physical and chemical properties and are of substantial interest for nano-electronics and sensor applications. ${ }^{1}$ Electrodeposition has established itself as a popular technique for the formation of such nanostructures. ${ }^{2}$ On the one hand, nanowire growth can be achieved by templates, for example by electrodeposition into porous membranes ${ }^{3-5}$ or by heterogeneous nucleation and growth at one-dimensional defects, such as steps. ${ }^{6-9}$ On the other hand, intrinsic anisotropies and kinetic effects can result in the spontaneous formation of one-dimensional nanostructures. The latter includes the well-known

\footnotetext{
${ }^{a}$ Christian-Albrechts-Universität zu Kiel, Olshausenstr. 40, D-24098 Kiel, Germany. E-mail: magnussen@ physik.uni-kiel.de

${ }^{b}$ York University, 4700 Keele Street, Toronto, Canada

$\dagger$ Electronic supplementary information (ESI) available: STM videos showing the dynamics of needle-like Bi islands. See DOI: 10.1039/c6fd00086j

$\$$ New address: Faculty of Engineering, Hokkaido University, Sapporo, Japan.
} 
electrochemical growth of whiskers and dendrites ${ }^{\mathbf{1 0}}$ as well as the lateral epitaxial growth of nanowires and strongly anisotropic crystallites on electrode surfaces. ${ }^{11-15}$ Here we investigate the microscopic growth mechanisms of such structures and the atomic-scale growth dynamics for the case of electrodeposited bismuth on Au electrodes.

Bi thin films and nanostructures have been receiving much attention in recent years owning to the unusual surface electronic structure of $\mathrm{Bi}^{16,17} \mathrm{Bi}$ exhibits strong spin-orbit coupling, making it of great interest to the field of topological insulators, and has electronic surface states that cross the Fermi level. Phenomena such as surface superconductivity, enhanced thermopower, and high electronic mobility have been observed, which are still under debate. As a consequence the behaviour of $\mathrm{Bi}$ nanowires and nanoribbons also strongly deviates from that of the bulk material and changes as a function of the nanostructure diameter and type. ${ }^{18-22}$ Furthermore, thin Bi films for potential applications in magnetic sensors, ${ }^{23,24}$ thermoelectric materials ${ }^{17,25}$ and spin filters ${ }^{26,27}$ depend strongly on the integrity of the microstructure. ${ }^{28}$ Bi thin films prepared by electrochemical deposition have been studied in recent years because of this method's ability to provide a better control of the thin film's microstructure, simple processing, such as room temperature operation at atmospheric pressure, and low costs. Electrochemical growth of crystalline Bi thin films has been demonstrated on $\mathrm{Au}(111)^{13,29}$ and $\operatorname{GaAs}(111),^{30}$ resulting in surface morphologies with needle-like and pyramidal islands, respectively. These deposits can be converted into highly ordered single-crystal Bi films by annealing. Similar needle-like morphologies were also found for Bi deposition from the gas phase on $\mathrm{Ag}(111),{ }^{31}$ $\operatorname{Si}(111)^{32,33}$ and graphite..$^{34,35}$

Detailed studies of the initial stage of Bi electrodeposition on $\mathrm{Au}(111)$ and $\mathrm{Au}(100)$ substrates were performed using in situ STM and in situ surface X-ray scattering measurements. ${ }^{13,36-41}$ Most of these studies focused on Bi underpotential deposition (UPD), revealing a similar sequence of Bi monolayer phases on both surfaces. With decreasing potential first commensurate Bi superstructures were found, followed by uniaxially-incommensurate quasi-hexagonal adlayers with high packing density. As shown by X-ray diffraction studies, bulk Bi deposition on $\mathrm{Au}(111)$ occurs in the form of an epitaxial film, in which the Bi(110) plane $\S$ is oriented parallel to the Au surface (see Fig. 1). ${ }^{29}$ An in situ STM study of the Bi growth behaviour was reported by Jeffrey et al. ${ }^{13}$ At low overpotential $\left(\approx-0.08 \mathrm{~V}_{\mathrm{SCE}}\right)$ the growth of these films was found to start with the formation of an isotropic Bi bilayer on the terraces, followed by the formation of nuclei at step edges and defect sites. These nuclei develop into highly anisotropic needle-like structures which are oriented with the long axis parallel to the Au surface and which grow quite rapidly. This needle growth mode is observed up to many hundreds of layers, resulting in a Bi film of increasing roughness. However, a clear mechanism of the nucleation and growth of these needles is still lacking, because of the high growth rate and the resulting difficulties in following this process using a conventional in situ STM.

\footnotetext{
$\S$ We employ throughout the paper the rhombohedral unit cell for crystallographic notation. In the discussion of literature where a hexagonal unit cell was employed, we have converted the notation for clarity. Specifically, we note that (110) and (111) in the rhombohedral system corresponds in the hexagonal notation to (012) and (001), respectively.
} 


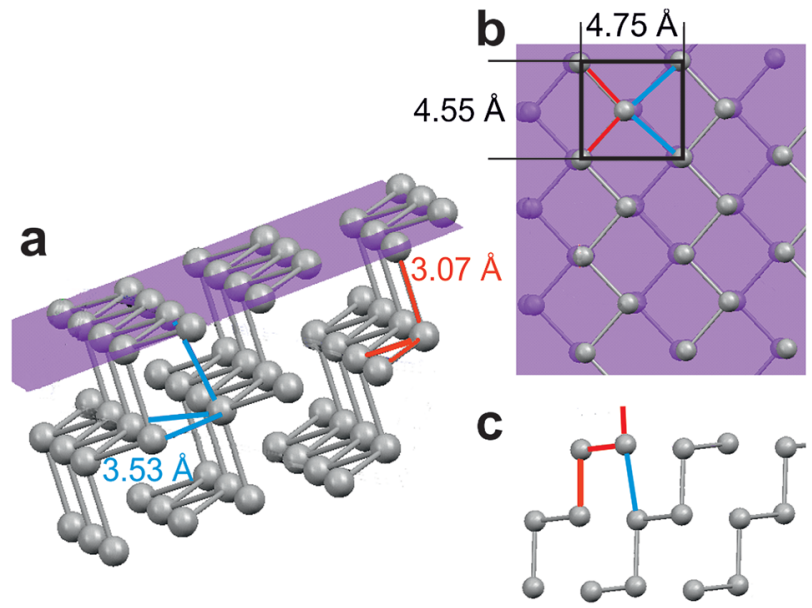

Fig. 1 (a) Structure of bulk bismuth, indicating short covalent (red) and long non-covalent (blue) bonding distances between neighbouring atoms. The shaded plane is the (110) plane. (b) In-plane structure and (c) side view of the (110) planes.

Such atomic-scale studies of fast dynamic processes at electrochemical interfaces have become feasible using in situ video STM, performed in constant height mode at scan rates of 10 to 30 images per second. ${ }^{42}$ In particular, video STM has been employed in the past for studies of electrochemical crystal growth and dissolution processes ${ }^{43-45}$ and of the dynamics of quasi one-dimensional nanostructures on electrode surfaces. ${ }^{46-49} \mathrm{Bi}$ deposition on $\mathrm{Au}$ single crystal electrodes in $\mathrm{HClO}_{4}$ solution is well suited for such video STM studies because (i) the $\mathrm{ClO}_{4}{ }^{-}$ anions are not specifically adsorbed on Bi and hence do not affect the Bi growth and (ii) the Bi electrodeposit exhibits a well-defined crystalline structure with extended terraces. In this paper we describe the structure and dynamics of $\mathrm{Bi}$ growth on $\mathrm{Au}(111)$ and $\mathrm{Au}(100)$. We especially focus on the origin of the pronounced anisotropy in this deposition process, manifesting in the formation of Bi needles and nanowires. The dynamic behaviour of kinks along the Bi needle edges is analysed quantitatively and interpreted taking the Bi lattice structure into account.

\section{Experimental}

The study was performed using the home-built in situ electrochemical video STM of the Kiel group. ${ }^{42}$ All images presented in this work were recorded at a rate of $10-$ 20 images per second in constant height mode. STM tips were etched from W-wire and coated with ethylene-vinyl acetate polymer, leaving the very end exposed. The tip and sample were independently kept under potentiostatic control.

The electrochemical cell consisted of a Kel-f cell with a charged palladium hydride electrode $(\mathrm{Pd}-\mathrm{H})$ as a reference, a $\mathrm{Pt}$ wire as a counter electrode and a $\mathrm{Au}(111)$ or $\mathrm{Au}(100)$ single crystal (orientation $\leq \pm 0.5^{\circ}$, Monocrystal Co.) as a working electrode. The $\mathrm{Pd}-\mathrm{H}$ electrode was charged prior to the experiment in a dilute sulfuric acid solution and exhibited a stable potential (ca. $-0.20 \mathrm{~V}$, vs. SCE) in the bismuth containing electrolytes. All the cell potentials reported in this 
work are given with respect to the saturated calomel electrode (SCE). The Au crystal was cleaned in suprapure sulfuric acid and rinsed in ultrapure water (Elga Lab water, 18.2 $\mathrm{M} \Omega$ resistance). The crystal was then flame annealed for several minutes and cooled down in air before being mounted in the video STM cell. The Bi electrolytes, containing 0.5 or $1 \mathrm{mM} \mathrm{Bi}{ }^{3+}$, were prepared by dissolving $\mathrm{Bi}_{2} \mathrm{O}_{3}$ (Alfa Aesar, 99.9995\%) in $0.1 \mathrm{M} \mathrm{HClO}_{4}$ (ultrapure grade, Seastar Chemicals Inc.) prepared using ultrapure water. In all experiments, the initial potential was chosen between 0.05 and $-0.08 \mathrm{~V}$, i.e. at a value more positive than the corresponding $\mathrm{Bi}^{3+} / \mathrm{Bi}$ Nernst potential, to avoid Bi bulk deposition. Subsequently, the potential was stepped down to more negative values in the overpotential deposition (OPD) regime to investigate the growth of bulk Bi.

\section{Results and discussion}

\section{Bismuth growth on $\mathrm{Au}(111)$}

In situ video STM experiments performed on $\mathrm{Au}(111)$ electrodes in the Bi OPD potential regime reveal the same needle growth behaviour observed using the conventional STM. When the cell potential is stepped to higher overpotentials $(<-0.10 \mathrm{~V})$, rapid formation of Bi multilayer deposits commences. Because the surface roughness in this stage of the growth process is initially high, the (constant-height mode) video-rate imaging is impeded and high-resolution images of the deposit cannot be obtained. However, 1 to 2 minutes after the onset of Bi bulk deposition the growth rate slows down and the characteristic Bi needle structure can be observed with atomic resolution (Fig. 2a). The Bi surface lattice is described by a pseudo-square structure with a prominent maximum at the corners of the unit cell and a weaker maximum slightly of center (shifted to the left of the unit cell center in the image in Fig. 2a). This structure is in full

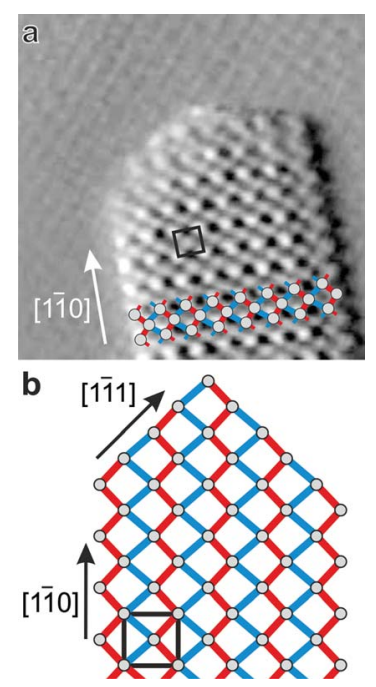

Fig. 2 (a) High-resolution in situ video STM image of the needle-like Bi deposit structure obtained on $A u(111)$ at $-0.19 \mathrm{~V}\left(46 \times 46 \AA^{2}\right)$. (b) Schematic model of the atomic structure of the Bi needles. 
agreement with the arrangement of Bi atoms in the Bi(110) plane (Fig. 1b). Within this plane parallel running zigzag chains of covalently bound $\mathrm{Bi}$ atoms are located. Each surface atom thus exhibits two covalent bonds of $3.07 \AA$ length (indicated in red) to atoms within the chain and two longer non-covalent bonds with $3.53 \AA$ bonding distance (indicated in blue) to atoms in the neighbouring chain. Very similar UHV-STM images were obtained on needle-like Bi deposits on $\mathrm{Ag}(111)$ and assigned to an identical structure. ${ }^{31}$ In some in situ video STM images obtained at larger bias only one of the atoms in the pseudo-square unit cell was resolved. Those observations strongly resemble high-resolution in situ STM images obtained in the previous study of $\mathrm{Bi}$ on $\mathrm{Au}(111)$ by Jeffrey et al. ${ }^{13}$ as well as in UHV-STM experiments of $\mathrm{Bi}(110)$ films on $\mathrm{Si}(111)-(7 \times 7) .^{32}$

The characteristic needle-like growth morphology can be clearly correlated with the anisotropic atomic structure of the Bi deposit. The Bi needles are oriented perfectly parallel to the $[1 \overline{1} 0]$ direction of the Bi lattice, i.e. along the direction of the covalently bound chains. Apart from a few kinks during needle growth and dissolution (see below), the needle edges along this direction are perfectly straight. Interestingly, each needle consists of an odd number of Bi rows, i.e. the needles are $(n+1 / 2)$ unit cells wide (with $n$ being a natural number). As illustrated schematically in Fig. 2b, these observations can be rationalised by an atomic arrangement where the needles are on both edges terminated by $\mathrm{Bi}$ chains. In this type of structure all Bi atoms at the needle edges are energetically stabilized by two covalent bonds to neighbouring edge atoms.

The needle-like Bi islands shown as examples in Fig. 2a grow on top of a smooth $\mathrm{Bi}(110)$ terrace (the pseudo-square unit cell of the latter surface is only faintly visible due to the much higher tunnelling distance in these constant height images). They are of monolayer height, which is in contrast to the needlelike Bi islands observed in UHV-STM studies of gas phase deposited Bi films. ${ }^{31-35}$ In the latter case the step heights typically corresponded to multiples of bilayers. This was attributed to a bilayer arrangement as in black phosphorous, where the out-of-plane dangling bonds of the Bi atoms pair up. Depending on the substrate the planes in the black phosphorous structure were found to be either flat or buckled. Our experimental observations suggest a bulk like (110) structure of the $\mathrm{Bi}$ deposit on $\mathrm{Au}(111)$ electrodes rather than a flat or buckled black phosphorous phase: first, no preference for films with an even number of Bi layers is found. Second, the two atoms in the $\operatorname{Bi}(110)$ surface unit cell appear significantly different in the STM images whereas no such difference in contrast was observed in high-resolution UHV-STM studies. ${ }^{31,32,34,50}$ For a bulk-like (110) film the two atoms in the unit cell are chemically non-equivalent, which could explain this difference in contrast. Specifically, the atoms in the center of the unit cell of Fig. 1b exhibit dangling bonds towards the electrolyte while those in the unit cell corners would have covalent bonds to the underlying Bi layer. This structural difference of the $\mathrm{Bi}(110)$ film in our study and under vacuum conditions may be caused by the termination of the dangling bonds by adsorbed species from the electrolyte. In view of the negative deposition potential the most likely candidate is adsorbed hydrogen, which readily binds to these sites. ${ }^{32,51-53}$ Evidence for the adsorption of hydrogen species, especially on Bi surfaces exhibiting covalent bonds, was also obtained in studies of hydrogen evolution. ${ }^{54}$ In contrast, the presence of other species, such as adsorbed anions or $\mathrm{OH}$, can be ruled out in this potential regime. ${ }^{55}$ 
The dynamics of the Bi islands reflects their anisotropic structure. While the long, atomically smooth steps along the [110] direction are very stable, the tips of the needle-like islands exhibit rough and highly mobile steps. In atomic resolution in situ video STM measurements these tips often appear blurry and change from image to image (see Fig. 3a and video 1 in the ESI $\dagger$ ), indicating fast attachment and detachment of Bi adatoms. This instability of the step edge atoms at the needle tips can be explained within the structural model proposed above. Because the zigzag chains are terminated at the tips, these atoms have a lower number of covalent bonds to neighbouring $\mathrm{Bi}$ atoms than the atoms at the [110]

a
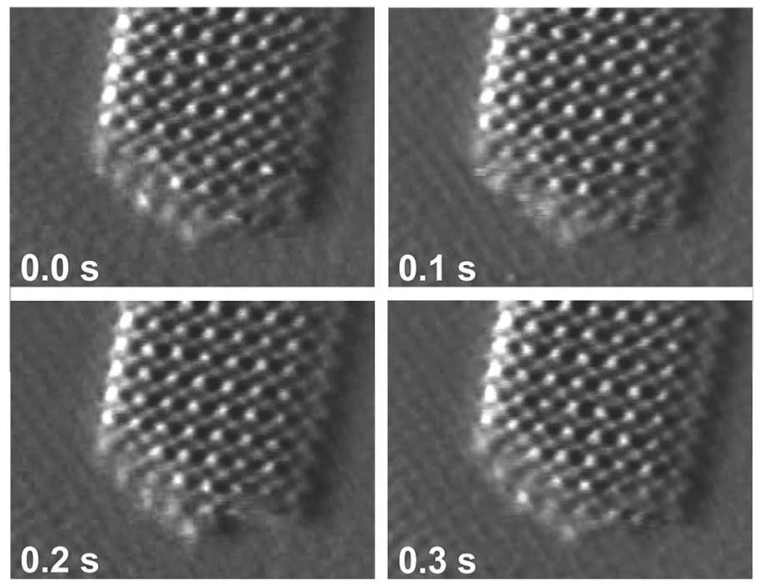

b
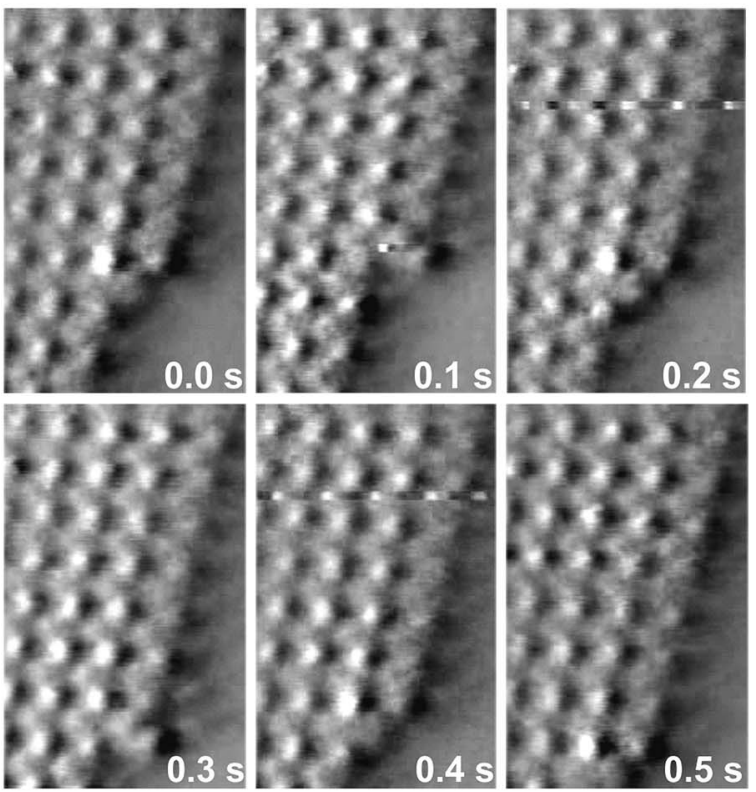

Fig. 3 Sequences of STM images of Bi deposits on $A$ (111), taken from videos recorded at $10 \mathrm{~Hz}$. (a) Fluctuations at the tip of a needle-like island, obtained at $-0.19 \mathrm{~V}\left(62 \times 51 \AA^{2}\right)$. (b) Kink propagation along the edge of a needle-like island, recorded at $-0.40 \mathrm{~V}\left(25 \times 38 \AA^{2}\right)$. 
oriented steps (see Fig. 2b). The weak tendency of the steps at the needle tips to orient roughly along the [111] orientation suggests that the step edge atoms can also be partly stabilized by the non-covalent bonds; however, this effect is much less pronounced than that of the covalent coordination.

The same tendencies manifest in video STM studies of the motion of kinks along the straight [110] oriented steps. These studies reveal that the kink motion proceeds via the addition or removal of two rows of Bi atoms in a zigzag fashion, i.e. via one-dimensional growth or dissolution of a chain of covalently bound $\mathrm{Bi}$ surface atoms along the island edge (see Fig. $3 \mathrm{~b}$ and video $2 \dagger$ ). Three types of kinks were observed in the STM videos, which are illustrated in Fig. 4: typically, kinks are of the type denominated as A or B in Fig. 4 and propagate along the step edge by alternating between these two states. Examples of these types of kinks can be observed e.g. in Fig. 3b. In more rare cases steps of the type $\mathrm{C}$ are observed in the images. Also in this case kink propagation occurs via subsequent changes between the type $\mathrm{C}$ and the type $\mathrm{B}$ state.

The microscopic growth rates found in these experiments are much lower than those which would be expected at the rather high overpotentials employed. We attribute this to geometric shielding by the STM tip, which is a well-known effect
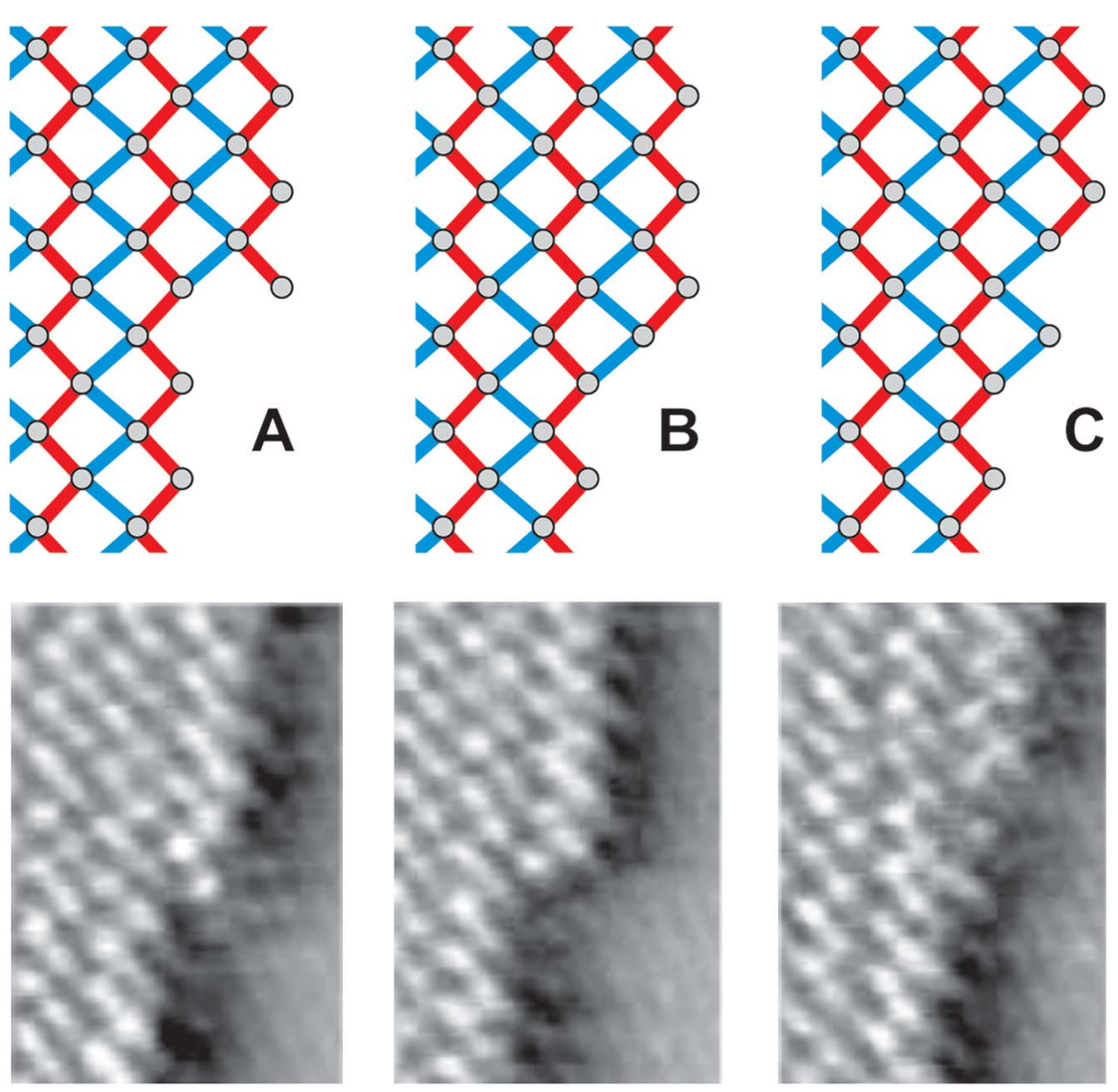

Fig. 4 Different types of kinks observed along the edges of Bi needles on $\mathrm{Au}(111)$ and corresponding examples, taken from an STM video. 
in in situ STM studies of electrodeposition processes. ${ }^{56}$ Nevertheless, the video STM observations of the structural fluctuations at the kinks provide interesting additional information on the Bi deposits. Because these fluctuations occur on the hundred millisecond time scale, the different kink configurations can be sampled with good statistics. According to our data, the different types of kinks are observed with very different probabilities. Based on an analysis of 750 kinks, we find the type B kinks with $81 \%$ probability, type A kinks with $16 \%$ probability and type $\mathrm{C}$ kinks with only $3 \%$ probability. Within the experimental errors no clear dependence on potential was found in the range -0.10 to $-0.64 \mathrm{~V}$. These statistical data reflect the different probabilities $p_{j}(j=\mathrm{A}, \mathrm{B}, \mathrm{C})$ for a kink to be in one of the three observed states, i.e. the different residence times of the kinks to be in one of these configurations. Because the studies were performed at low kink propagation rates and thus are close to equilibrium, these statistical data allow the calculation of the relative energy difference $\Delta E_{i j}$ between these kinks via the Boltzmann equation $\Delta E_{i j}=-k_{\mathrm{B}} T \ln \left(p_{i} / p_{j}\right)$. Using the statistical data obtained in the video STM measurements (performed at $T=298 \mathrm{~K}$ ) one obtains an increase in energy of $\Delta E_{\mathrm{AB}}=42 \pm 3 \mathrm{meV}$ of the type $\mathrm{A}$ and of $\Delta E_{\mathrm{CB}}=86 \pm 7 \mathrm{meV}$ of the type $\mathrm{C}$ kinks as compared to the type B kinks, respectively.

We interpret these results in terms of the different coordination of the $\mathrm{Bi}$ atoms at the kink in the three different states. In the type A kinks the Bi atoms at the kink are missing two non-covalent bonds to neighbouring atoms as compared to the type B kinks; type $\mathrm{C}$ kinks are even lacking two covalent bonds. Taken this into account, the energy differences at a first glance seem smaller than expected from the Bi cohesive energy $\left(2.16 \mathrm{eV}\right.$ per atom $\left.{ }^{57}\right)$ or the Bi-Bi bond energy $(2.08 \mathrm{eV}$ (ref. 58)). However, the energy balance may be strongly affected by the presence of coadsorbates. Recent density functional theory calculations for two-dimensional Bi nanoribbons with similar covalently bound Bi chains along the edges found strong modification of the geometric and electronic structure by hydrogen adsorption at the dangling bonds of the terminating Bi atoms. ${ }^{51-53}$ Hydrogen adsorption energies in the range of $2.2 \mathrm{eV}$ were reported. ${ }^{52}$ Similar behaviour may be expected in the electrochemical environment and may significantly reduce the kink energies.

\section{Bismuth growth on $\mathrm{Au}(100)$}

Similar in situ video STM studies of Bi electrodeposition were performed on $\mathrm{Au}(100)$ electrodes. In contrast to the structure of the Bi UPD adlayer on $\mathrm{Au}(100)$, which has been characterized in detail by STM and X-ray surface diffraction, ${ }^{36,40}$ the initial stages of Bi bulk electrodeposition have not been reported before. Highresolution video STM images recorded in the overpotential range (Fig. 5a) find a lattice structure that clearly differs from that of the UPD layer or of the Bi(110) film observed on $\mathrm{Au}(111)$. In particular, these Bi deposits exhibit a much more open atomic structure, which is incompatible with $\mathrm{Bi}(110)$ or the $\mathrm{c}(p \times 2) \mathrm{Bi}$ UPD layer. The observed surface structure is described by a rectangular unit cell with one prominent and one weaker maximum that form alternating rows. Upon assigning both of these to Bi surface atoms, a (pseudo-) hexagonal arrangement results. The interatomic distances are in good agreement with those in an atomic layer of the (111) plane of bulk Bi (shown in the model in Fig. 5b). In this plane the $\mathrm{Bi}$ atoms form hexagonally ordered bilayers, interlinked by covalent bonds. This 


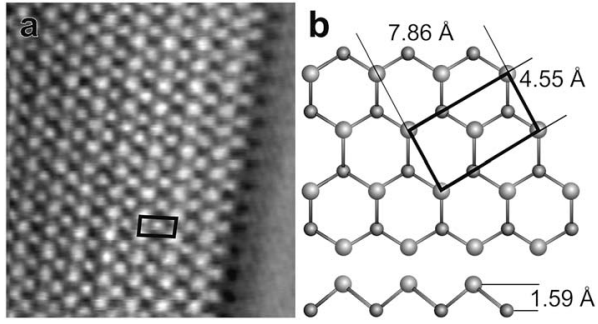

Fig. 5 (a) High-resolution in situ video STM image of the Bi deposit structure obtained on $\mathrm{Au}(100)$ at $-0.17 \mathrm{~V}\left(75 \times 60 \AA^{2}\right)$. (b) Model of the atomic structure of the Bi(111) plane, showing the first (light grey) and second (dark grey) Bi layer. In the bottom part a side view of the bilayer arrangement in this plane is shown.

assignment assumes that only the topmost $\mathrm{Bi}(111)$ layer is visible in the STM images, which is likely in view of the $1.59 \AA$ spacing to the underlying second layer (see Fig. 5b, bottom).

$\mathrm{Bi}(111)$ is the natural cleavage plane of bismuth and under UHV conditions the preferred orientation in epitaxial growth. ${ }^{16}$ The surface atoms in $\mathrm{Bi}(111)$ have a lower number of non-covalent long bonds to neighbouring atoms as compared to those in the $\mathrm{Bi}(110)$ surface, but - in contrast to the latter - do not exhibit dangling bonds. For thick epitaxial Bi films formed by electrodeposition on $\mathrm{Au}(100)$ a (110) orientation has been found. ${ }^{59}$ However, $\mathrm{Bi}(110)$ and $\mathrm{Bi}(111)$ films are similar in energy and structural transitions between them have been found in ultrathin epitaxial films. ${ }^{32,60}$ It therefore seems possible that a $\mathrm{Bi}(111)$ structure is epitaxially stabilized in the very thin films studied in this work (typically consisting of only a few layers).

Although a Bi(111) film would account for the observed inter-atomic spacings of the surface atoms, it cannot explain the row-like modulation of the structure. The latter would not be expected for true Bi(111) films and was not observed in previous STM studies of (111) oriented Bi films on Si(111)..$^{32,60}$ Most likely, these rows result from epitaxy to the underlying Au substrate lattice. This is supported by observations of the deposit morphology on larger length scales, which show that the rows in different $\mathrm{Bi}$ islands are arranged at well-defined angles of $90^{\circ}$ with respect to each other (see below). Interestingly, the $7.86 \AA$ spacing between nextnearest neighbour atoms of the topmost $\mathrm{Bi}(111)$ layer differs by only $3.7 \%$ from $2 a_{\mathrm{Au}}$ (with $a_{\mathrm{Au}}=4.08 \AA$ being the Au lattice constant). Furthermore, the Bi spacing in the perpendicular direction is only $11.5 \%$ larger than $a_{\mathrm{Au}}$. Minor deformation of the $\mathrm{Bi}(111)$ bilayer lattice thus allows it to transform into a structure that is fully commensurate by the $\mathrm{Au}(100)$ lattice and can be described by a $(\sqrt{ } 2 \times 2 \sqrt{2}) \mathrm{R}^{\circ} 5^{\circ}$ superstructure. In this structure half of the second layer Bi atoms would occupy the energetically preferred fourfold-hollow sites while the remaining half would reside on top sites, explaining the vertical modulation. The length of the covalent $\mathrm{Bi}-\mathrm{Bi}$ bonds can be preserved in this arrangement by a small vertical relaxation of the bilayer distance; the bond angles likewise will only be slightly distorted. Such a commensurate structure may significantly reduce the $\mathrm{Bi}-\mathrm{Au}$ interface energy and by this stabilize ultrathin $\operatorname{Bi}(111)$ films. For thicker deposits the epitaxial stress associated with this structure should drive a structural transition, apparently resulting in a $\mathrm{Bi}(110)$ film. 
Observations of the growth morphology and dynamics support that the ultrathin Bi deposits on $\mathrm{Au}(100)$ differ structurally from the Bi(110) film found on $\mathrm{Au}(111)$. First, the observed $\mathrm{Bi}$ islands are rectangular in shape rather than needlelike. Similar to the latter they exhibit relatively straight edges which are preferentially oriented along the direction of the atomic rows. Typically, the islands are terminated by one of the rows that appear to be of lower apparent height in the STM images (see Fig. 5a). However, compared to the Bi deposits on $\mathrm{Au}(111)$ these steps usually exhibit a much larger number of kinks that often are multiple rows wide.

The typical structure and dynamic behaviour of these kinks is illustrated in Fig. 6. Kinks in the step are usually one unit cell (or a multiple thereof) wide, i.e. consisting of two Bi rows. They are predominantly terminated by an atomically smooth edge (see e.g. Fig. 6, $0.0 \mathrm{~s}$ ) or an additional atom in the kink corner (Fig. 6, $0.1 \mathrm{~s}$ ), resembling the B and C type kinks illustrated in Fig. 4. However, the kinks propagate in a much more irregular fashion along the step edges than for Bi on $\mathrm{Au}(111)$. In particular, single rows that extend several atoms away from the kink are often formed transiently during island growth or dissolution (Fig. 6, 0.2-0.3 s). Such structures were never observed in videos of kink propagation along the edges of the needle-like $\mathrm{Bi}(110)$ islands, where they would correspond to atoms that are bound to the step only by long non-covalent bonds (see model of $\mathrm{C}$ type kinks in Fig. 4) and thus are energetically very unfavourable. In contrast, these observations can be easily reconciled with a $\mathrm{Bi}(111)$ deposit. Because all covalent bonds of the Bi surface atoms in the (111) plane are to atoms in the underlying second layer, the different kink configurations should be similar in energy.

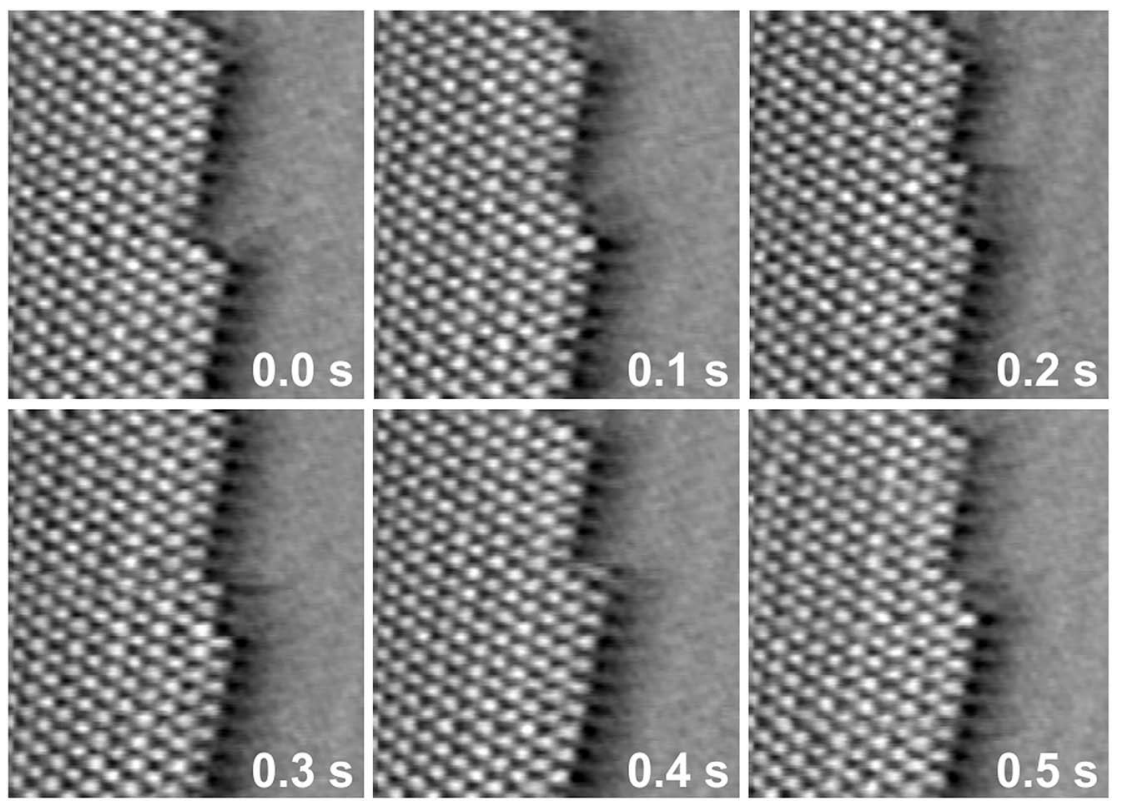

Fig. 6 Sequences of STM images of Bi deposits on Au(100), taken from a video recorded at $10 \mathrm{~Hz}$ and a potential of $-0.17 \mathrm{~V}$. The images show kink propagation along the island edge $\left(75 \times 70 \AA^{2}\right)$. 
A difference between the high and the low atomic rows could in this case only come from the different epitaxial relationship with respect to the substrate lattice, which should be a more minor effect.

In addition to these two-dimensional islands spontaneous formation of truly onedimensional nanowires was observed for $\mathrm{Bi}$ deposition on the $\mathrm{Au}(100)$ surface (Fig. 7). These nano-wires are of well-defined width and can be several tens of nanometers long. They grow predominantly away from the edges of the Bi islands, following the directions of the atomic rows (see Fig. 7c). Furthermore, structural fluctuations are observed in which Bi islands are converted into nanowires and vice versa. Often the nanowires form arrays with well-defined spacings that are exactly
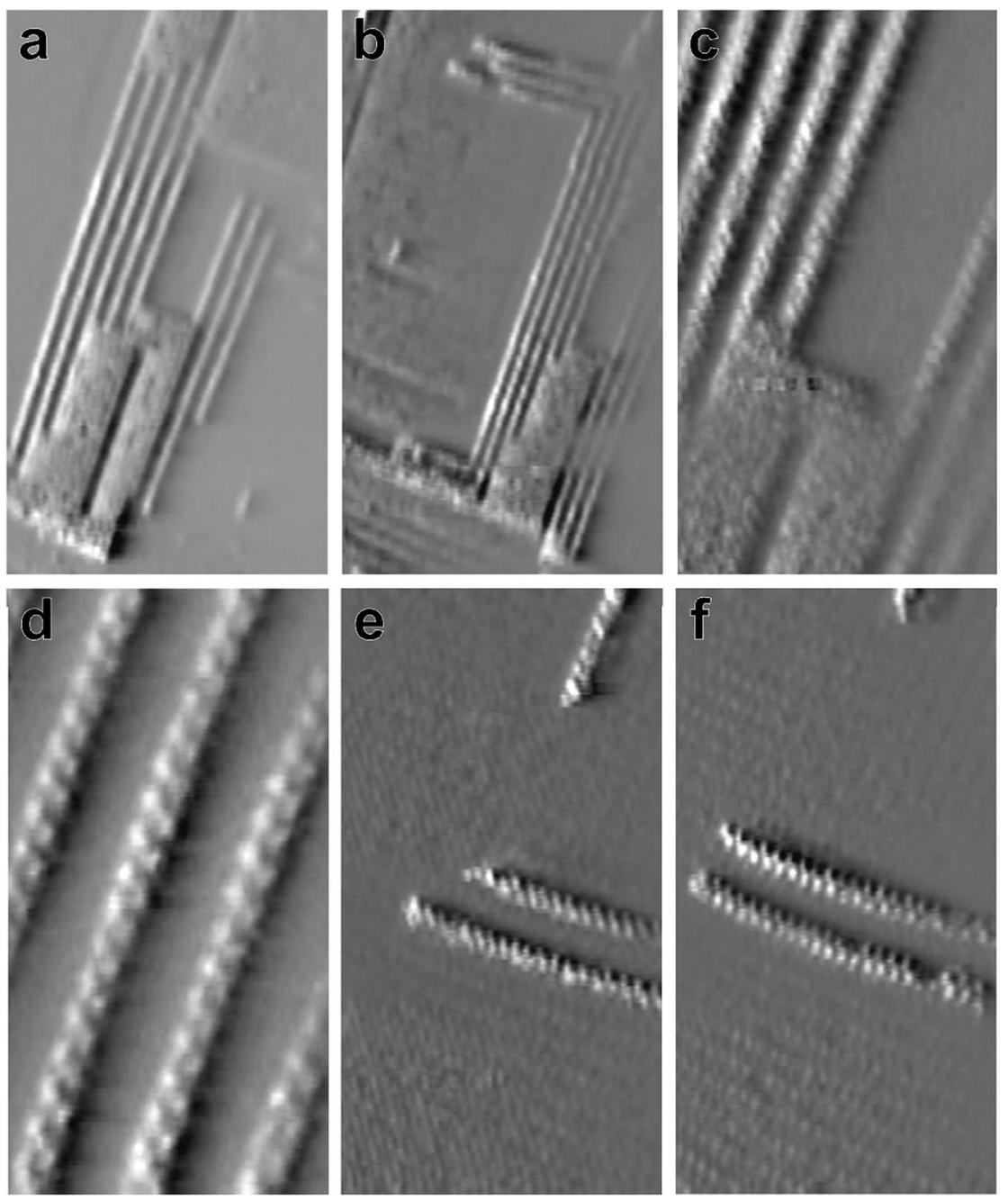

Fig. 7 (a) In situ video STM images of Bi nanowires on Au(100), recorded at $-0.26 \mathrm{~V}$. (a) Bi islands and arrays of nanowires $\left(165 \times 330 \AA^{2}\right)$. (b) Same surface area as in (a) recorded 25.5 s later $\left(260 \times 460 \AA^{2}\right)$. (c) Center of the area in (a) showing the atomic rows on islands and nanowires $\left(75 \times 130 \AA^{2}\right)$. (d) High-resolution image of the nanowire array $\left(32 \times 60 \AA^{2}\right)$. (e and f) Images of isolated nanowires recorded at a time interval of $1.0 \mathrm{~s}\left(85 \times 170 \AA^{2}\right)$. 
five times the distance between the atomic rows in the Bi islands (Fig. 7a-d). In more rare cases freestanding nanowires are also observed (Fig. 7e and f). The nanowires slowly grow with time, both in length and number, as can be seen in the image in Fig. $7 \mathrm{~b}$, recorded $25.5 \mathrm{~s}$ after the image in Fig. $7 \mathrm{a}$ in the same surface area. In addition, rapid fluctuations are found, especially for isolated free-standing nanowires. However, these fluctuations may be (at least partly) induced by the STM tip.

High-resolution images of the nanowires (Fig. 7d-f) reveal that they consist of a zigzag chain of atoms. As visible in the images in which the underlying Bi film is atomically resolved (Fig. 7e and f), these chains are commensurate to the Bi lattice. That is, the nanowires are exactly one of the rectangular unit cells of the Bi film wide and are oriented in the direction of the shorter unit cell vector. In areas where the nanowires are $90^{\circ}$ rotated (e.g. in the upper right corner of Fig. 7e and f) the atomic rows in the underlying Bi substrate also run in the perpendicular direction. These observations indicate that the nanowires grow epitaxially on the Bi bulk deposit and, most likely, are structurally related to the latter. However, it is currently unclear what stabilises the unique one-dimensional morphology of these structures.

\section{Conclusions}

As demonstrated by these in situ video STM studies, Bi electrodeposition exhibits a rich microscopic growth behaviour, reflecting its unique material properties. A central aspect governing the deposit morphology and dynamic is the presence of short bonds with pronounced covalent character. These bonds have already been found to determine the epitaxial growth of ultrathin Bi films under UHV conditions as well as to strongly influence the electronic surface and edge states of onedimensional Bi nanostructures - a topic of great current interest. Our observations indicate that the presence of covalent $\mathrm{Bi}-\mathrm{Bi}$ bonds also governs epitaxial growth in an electrochemical environment. Specifically, the formation of the needle-like Bi deposits on $\mathrm{Au}(111)$ found in previous STM studies can be clearly rationalised by the structural anisotropy in the deposit caused by these bonds. The latter interlink the atoms in the $\operatorname{Bi}(110)$ surface in the form of one-dimensional chains that define the needle direction. Growth and dynamic fluctuations occur predominantly at the end of these chains.

While thick Bi electrodeposits grow in the form of a Bi(110) film on $\mathrm{Au}(111)$ as well as on $\mathrm{Au}(100)$, video STM investigations of ultrathin deposits reveal a very different structure and growth morphology. As indicated by these data, the structure of ultrathin Bi electrodeposits may be strongly affected by epitaxial effects. On the basis of our structural observations we suggested the formation of a $\mathrm{Bi}(111)$ like film on $\mathrm{Au}(100)$, which is commensurate to the square substrate lattice. Independent confirmation of this deposit structure, e.g. by surface X-ray diffraction studies, would be highly desirable. Particularly interesting is the surprising observation of truly one-dimensional Bi nanowires on this surface. Structures of this type have not been reported in previous studies of Bi deposits, including deposits formed under vacuum conditions. Although their origin is currently unclear, they represent unique nanostructures with potentially interesting novel properties. In particular, one-dimensional Bi nanostructures are of great current interest in the field of topological insulators. The epitaxial electrodeposition of Bi may thus offer attractive new possibilities for the formation of such structures. 


\section{Acknowledgements}

We gratefully acknowledge financial support by the Deutsche Forschungsgemeinschaft via MA 1618/11 and MA 1618/15, NSERC for a Discovery Grant and a Canada Research Chair (S. M.), a fellowship for S. M. by the Alexander von HumboldtStiftung, and field trip funds from York University for S.-H. L.

\section{Notes and references}

1 Y. Xia, P. Yang, Y. Sun, Y. Wu, B. Mayers, B. Gates, Y. Yin, F. Kim and H. Yan, Adv. Mater., 2003, 15, 353.

2 G. Staikov, in Electrocrystallization in nanotechnology, Wiley-VCH, Weinheim, 2007.

3 T. R. Kline, M. Tian, J. Wang, A. Sen, M. W. H. Chan and T. E. Mallouk, Inorg. Chem., 2006, 45, 7555.

4 H. Schloerb, V. Haehnel, M. S. Khatri, A. Srivastav, A. Kumar, L. Schultz and S. Faehler, Phys. Status Solidi B, 2010, 247, 2364.

5 U. S. Mohanty, J. Appl. Electrochem., 2011, 41, 257.

6 R. M. Penner, J. Phys. Chem. B, 2002, 106, 3339.

7 R. M. Penner, Mod. Aspects Electrochem., 2009, 45, 175.

8 S. Morin, A. Lachenwitzer, O. M. Magnussen and R. J. Behm, Phys. Rev. Lett., 1999, 83, 5066.

9 X. Xiao, M. Nielinger and H. Baltruschat, Electrochim. Acta, 2003, 48, 3093.

10 J. K. Chang, C. M. Wu and I. W. Sun, J. Mater. Chem., 2010, 20, 3729.

11 F. Möller, O. M. Magnussen and R. J. Behm, Phys. Rev. Lett., 1996, 77, 3165.

12 S. Strbac, O. M. Magnussen and R. J. Behm, Phys. Rev. Lett., 1999, 83, 3246.

13 C. A. Jeffrey, D. A. Harrington and S. Morin, Surf. Sci., 2002, 512, L367.

14 S. Takakusagi, K. Kitamura and K. Uosaki, J. Phys. Chem. C, 2008, 112, 3073.

15 R. Wen, G. B. Pan and L. J. Wan, J. Am. Chem. Soc., 2008, 130, 12123.

16 P. Hofmann, Prog. Surf. Sci., 2006, 81, 191.

17 T. E. Huber, K. Owusu, S. Johnson, A. Nikolaeva, L. Konopko, R. C. Johnson and M. J. Graf, J. Appl. Phys., 2012, 111, 043709.

18 L. Li, Y. Yang, X. Fang, M. Kong, G. Li and L. Zhang, Solid State Commun., 2007, 141, 492.

19 M. Tian, J. Wang, Q. Zhang, N. Kumar, T. E. Mallouk and M. H. W. Chan, Nano Lett., 2009, 9, 3196.

20 K. Lee, S. Lee, S. N. Holmes, J. Ham, W. Lee and C. H. W. Barnes, Phys. Rev. B: Condens. Matter Mater. Phys., 2010, 82, 245310.

21 J. Kim, S. Lee, Y. M. Brovman, M. G. Kim, P. Kim and W. Lee, Appl. Phys. Lett., 2014, 104, 043105.

22 M. Tian, J. Wang, W. Ning, T. E. Mallouk and M. H. W. Chan, Nano Lett., 2015, 15, 1487.

23 F. Y. Yang, K. Liu, K. M. Hong, D. H. Reich, P. C. Searson and C. L. Chien, Science, 1999, 284, 1335.

24 F. Y. Yang, K. Liu, C. L. Chien and P. C. Searson, Phys. Rev. Lett., 1999, 82, 3328.

25 Z. Zhang, X. Sun, M. S. Dresselhaus, J. Y. Ying and J. Heremans, Phys. Rev. B: Condens. Matter Mater. Phys., 2000, 61, 4850.

26 Y. Koroteev, G. Bihlmayer, J. E. Gayone, E. V. Chulkov, S. Blügel, P. M. Echenique and P. Hofmann, Phys. Rev. Lett., 2004, 93, 046403. 
27 T. Hirahara, T. Nagao, I. Matsuda, G. Bihlmayer, E. V. Chulkov, Y. Koroteev, P. M. Echenique, M. Saito and S. Hasegawa, Phys. Rev. Lett., 2006, 97, 146803.

28 B. O'Brien, M. Plaza, L. Y. Zhu, L. Perez, C. L. Chien and P. C. Searson, J. Phys. Chem. C, 2008, 112, 12018.

29 C. A. Jeffrey, S. H. Zheng, E. Bohannan, D. A. Harrington and S. Morin, Surf. Sci., 2005, 600, 95.

30 Z. L. Bao and K. L. Kavanagh, Appl. Phys. Lett., 2006, 88, 063708.

31 H. L. Zhang, W. Chen, X. S. Wang, J. Yuhara and A. T. S. Wee, Appl. Surf. Sci., 2009, 256, 460.

32 T. Nagao, J. T. Sadowski, M. Saito, S. Yaginuma, Y. Fujikawa, T. Kogure, T. Ohno, Y. Hasegawa, S. Hasegawa and T. Sakurai, Phys. Rev. Lett., 2004, 93, 105501.

33 S. Yaginuma, T. Nagao, J. T. Sadowski, M. Saito, K. Nagaoka, Y. Fujikawa, T. Sakurai and T. Nakayama, Surf. Sci., 2007, 601, 3593.

34 P. J. Kowalczyk, O. Mahapatra, D. N. McCarthy, W. Kozlowski, Z. Klusek and S. A. Brown, Surf. Sci., 2011, 605, 659.

35 P. J. Kowalczyk, O. Mahapatra, S. A. Brown, G. Bian, X. Wang and T. C. Chiang, Nano Lett., 2013, 13, 43.

36 S. H. Zheng, K. Krug, F. Golks, D. Kaminski, S. Morin and O. M. Magnussen, J. Electroanal. Chem., 2010, 649, 189.

37 C.-H. Chen and A. A. Gewirth, J. Am. Chem. Soc., 1992, 114, 5439.

38 C. Chen, K. D. Kepler, A. A. Gewirth, B. M. Ocko and J. Wang, J. Phys. Chem., 1993, 97, 7290.

39 K. Tamura, B. M. Ocko, J. X. Wang and R. R. Adzic, J. Phys. Chem. B, 2002, 106, 3896.

40 M. Hara, Y. Nagahara, S. Yoshimoto, J. Inukai and K. Itaya, J. Electrochem. Soc., 2004, 151, E92.

41 M. Nakamura, N. Sato, N. Hoshi and O. Sakata, Langmuir, 2010, 26, 4590.

42 L. Zitzler, B. Gleich, O. M. Magnussen and R. J. Behm, Proceedings of the Electrochemical Society, 2000, vol. 99-28, p. 29.

43 O. M. Magnussen, L. Zitzler, B. Gleich, M. R. Vogt and R. J. Behm, Electrochim. Acta, 2001, 46, 3725.

44 O. M. Magnussen, W. Polewska, L. Zitzler and R. J. Behm, Faraday Discuss., 2002, 121, 43.

45 W. Polewska, R. J. Behm and O. M. Magnussen, Electrochim. Acta, 2003, 48, 2915.

46 M. Labayen and O. M. Magnussen, Surf. Sci., 2004, 573, 128.

47 M. Labayen, C. Haak and O. M. Magnussen, Phys. Rev. B: Condens. Matter Mater. Phys., 2005, 71, 241409-241411.

48 H. Matsushima, A. Taranovskyy, C. Haak, Y. Gründer and O. M. Magnussen, J. Am. Chem. Soc., 2009, 131, 10362.

49 H. Matsushima, C. Haak, A. Taranovskyy, Y. Gründer and O. M. Magnussen, Phys. Chem. Chem. Phys., 2010, 12, 13992.

50 Y. Lu, W. Xu, M. Zeng, G. Yao, L. Shen, M. Yang, Z. Luo, F. Pan, K. Wu, T. Das, P. He, J. Jiang, J. Martin, Y. P. Feng, H. Lin and X. S. Wang, Nano Lett., 2015, 15, 80.

51 Z. F. Wang, L. Chen and F. Liu, Nano Lett., 2014, 14, 2879.

52 L. Chen, G. Cui, P. Zhang, X. Wang, H. Liu and D. Wang, Phys. Chem. Chem. Phys., 2014, 16, 17206. 
53 D. Wang, L. Chen, H. Liu, X. Wang, G. Cui, P. Zhang, D. Zhao and S. Ji, Phys. Chem. Chem. Phys., 2015, 17, 3577.

54 K. Lust, E. Perkson and E. Lust, Russ. J. Electrochem., 2000, 36, 1257.

55 X. Li and A. A. Gewirth, J. Am. Chem. Soc., 2003, 125, 7086.

56 O. Skylar, T. H. Treutler, N. Vlachopoulos and G. Wittstock, Surf. Sci., 2005, 597, 181.

57 C. Kittel, in Introduction to solid state physics, Wiley, NY, 3rd edn, 1968.

58 D. R. Lide, in CRC handbook of chemistry and physics, CRC Press, Boca Raton, 73th edn, 2000.

59 S. H. Zheng, PhD thesis, York University, 2011.

60 T. Nagao, S. Yaginuma, M. Saito, T. Kogure, J. T. Sadowski, T. Ohno, S. Hasegawa and T. Sakurai, Surf. Sci., 2005, 590, L247. 